\section{Metastatische Kalzinose der Lunge}

Pulmonale Verkalkungen sind ein häufiger Thoraxbefund. Sie finden sich bei pulmonalen und nicht-pulmonalen Erkrankungen, sind Ausdruck abgeheilter bzw. progredienter Prozesse. Ihr Erscheinungsbild erlaubt Rückschlüsse auf Ätiologie, Verlauf und evtl. Therapieerfolg. Grundsätzlich wird zwischen Verkalkungen vom „dystrophen“, vom „benigne metastatischen“ und vom „malignen Typ“ unterschieden.

Die Verkalkungen vom dystrophen Typ sind am häufigsten und basieren auf Ablagerungen in pathologisch verändertem, meist nekrotischem Gewebe z.B. nach Infektionen. Die benignen Verkalkungen haben ihre Ursache in Störungen des Kalzium-Phosphat-Stoffwechsels, die zum Einbau von Kalziumsalzen in gesundes Gewebe führen. Maligne Verkalkungen dagegen entstehen durch die Fähigkeit maligner Tumorzellen, Kalziumsalze zu metabolisieren und einzulagern.

Die folgende Kasuistik berichtet über ausgedehnte benigne metastatische Verkalkungen (BMV) der Lunge bei einer Patientin mit sekundärem Hyperparathyreoidismus (SHPT) nach Niereninsuffizenz und Nierentransplantation.

\section{Fallbericht}

Eine 48jährige Patientin erhielt nach 4 Jahren dialysepflichtiger Niereninsuffizienz ein Nierentransplantat (11/97). Vor der Operation angefertigte konventionelle Röntgenaufnahmen des Thorax zeigten beidseits basal diskrete Verschattungen. Ein halbes Jahr nach Transplantation entwickelte die Patientin unter Cortisontherapie zunehmende Dyspnoe, Husten, Leistungsabfall und einen CRP-Anstieg auf 6,31 mg/dl (Norm $(0,04-0,5 \mathrm{mg} / \mathrm{dl})$. Die Beschwerden besserten sich unter Antibiose nur gering. Im Röntgenthorax (Abb.1 vom 21.10.1998) fanden sich beidseits basal betonte, klein- bis grobfleckige, teils konfluierende Verschattungen, die als Pneumonie gedeutet wurden. Nachdem

Fortschr Röntgenstr 2000; 172: 487-488 (c) Georg Thieme Verlag Stuttgart · New York ISSN 1438-9029

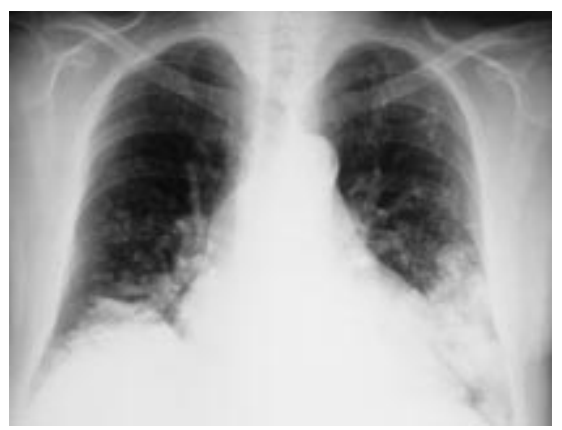

Abb. 1 Die Thoraxaufnahme p.a. vom 21.10.1998 zeigt beidseits basal betonte, klein- bis grobfleckige, teils konfluierende Verschattungen.

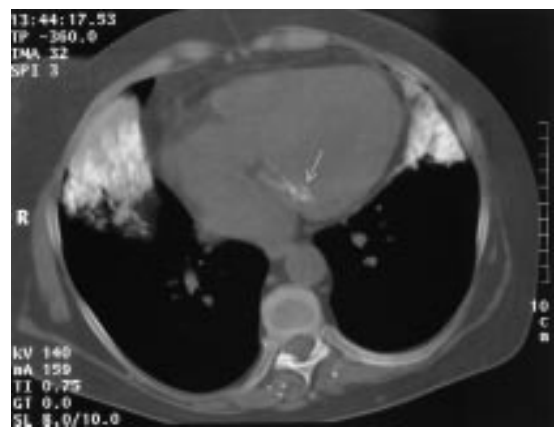

Abb. 2 Die native Computertomographie vom 23.10.1998 zeigt ausgedehnte parenchymatöse Verkalkungen vor allem des Mittellappens und des Lingulasegmentes, mit Dichtewerten bis über $1000 \mathrm{HE}$, sowie deutliche Kalkablagerungen an der Mitralklappe (Pfeil).

eine Bronchiallavage und eine transbronchiale Biopsie keinen Anhalt für eine Pneumonie ergaben, zeigte eine CT des Thorax (Abb. 2 vom 23.10.98) ausgedehnte parenchymatöse Verkalkungen vor allem im Mittellappen und der Lingula sowie deutlich Verkalkungen der Mitralklappe.

Nach Nierentransplantation bestand laborchemisch ein ausgeprägter sekundärer Hyperparathyreoidismus: Parathormon (PTH) 641,0 ng/l (Norm: 12,0$72,0 \mathrm{ng} / \mathrm{l})$, Kalzium 2,84 mmol/l (2,03$2,6 \mathrm{mmol} / \mathrm{l})$, Phosphat $2,66 \mathrm{mmol} / \mathrm{l}$ $(0,87-1,45 \mathrm{mmol} / \mathrm{l})$, Kalzium/PhosphatProdukt 7,55 mmol/l $(<6,0 \mathrm{mmol} / \mathrm{l})$. Die Lungenfunktion ergab eine geringgradige periphere Obstruktion mit Teilrestriktion. In der CCT-Untersuchung fanden sich diskrete Pallidumverkalkungen linksseitig. Des weiteren bestanden ausgeprägte Gefäßverkalkungen der Koronarien und der Aorta sowie der Extremitätenarterien.

11/98 wurde eine Parathyreoidektomie mit Autotransplantation eines Epithelkörperchens in den Unterarm durchgeführt. In den Wochen postoperativ normalisierten sich das PTH und unter medikamentöser Therapie auch die Werte für Kalzium und Phosphat. Die pulmonalen Verkalkungen haben im Thoraxbild seit der Parathyreoidektomie nicht mehr relevant zugenommen.

\section{Diskussion}

Kuhlman J.E. et al. (Radiology 1989; 173: 459 - 460) beschreiben, daß ausgedehntere Kalzifikationen oft erst post mortem in Zusammenschau mit dem Sektionsbefund auf dem Röntgen-Thoraxbild als solche diagnostiziert werden, obwohl sie aufgrund von Ausdehnung, Dichte und Verlauf als solche zu erkennen wären.

Histologisch sind BMV in den Alveolarsepten lokalisiert, können jedoch auch pulmonale Arterien und Bronchialwände betreffen (Murris-Espin M. et al., Eur Resp J 1997; 10: 1925-1927). Schwere Kalzifikationen gehen oft mit einer interstitiellen Fibrose einher („Bims-“ oder „Tuffsteinlunge“).

Der Verkalkungsprozeß ist abhängig von laborchemischen Parametern wie z.B. $\mathrm{Ca} 2+$ und $\mathrm{PO}_{4}{ }^{3}$ (Murris-Espin M. et al.). Romagnoli M. et al. (Eur Resp J 1997; 10: 958 - 960) beschrieben jedoch auch ausgedehnte BMV ohne Veränderungen dieser Laborparameter.

Häufigster Grund für pulmonale BMV sind die chronische Niereninsuffizienz und/oder ein primärer, sekundärer oder tertiärer Hyperparathyreoidismus von langer Dauer (Kuhlman J.E. et al.), wie sie auch bei unserer Patientin vorlagen. Nach Murris-Espin M. et al. finden sich BMV in Autopsien von Dialysepatienten zu $60-90 \%$.

Die wichtigste klinische Differentialdiagnose sind Pneumonien, da sie ähnliche Symptome machen (Husten, Dyspnoe). BMV sind meist symptomärmer und durch Labor und klinischen Verlauf (Ansprechen auf Antibiotika) zu differenzieren. Bei ausgedehnteren Befunden sind Reizhusten, Dyspnoe und objektiv nachweisbare restriktive Ventilationsstörun- 
gen beschrieben (Oursin C. et al., Radiologe 1992; 32: 77-79). Sie können zu Lungenfibrose, Cor pulmonale und respiratorischer Insuffizienz führen.

Pneumonien sind eher basal lokalisiert und zeigen wechselnde Verteilung, Dichte und Konfiguration. Prädilektionsstellen für pulmonale BMV sind aufgrund des höheren Ventilations-/ Perfusions-Verhältnisses die oberen Lungenabschnitte. Dort führt der erhöhte $\mathrm{O}_{2}$ - und erniedrigte $\mathrm{CO}_{2}$-Gehalt zu einem pH-Anstieg auf ca. 7,51, verglichen mit ca. 7,39 in den basalen Abschnitten, und dies begünstigt nach Oursin C. et al. das Ausfällen von Kalziumsalzen.

Da die Verkalkungen in unserem Fall für BMV untypisch in den basalen Lungenabschnitten lokalisiert sind, sind differentialdiagnostisch auch Verkalkungen vom dystrophen Typ, die sich in einem durch eine basale Pneumonie vorgeschädigtem Gewebe entwickelt haben, in Betracht zu ziehen.

Nach Henk et al. (Radiologe 1996; 36: 534 -542) führen auch kardiovaskuläre Ursachen häufig zu pulmonalen Verkalkungen. Bei einer Mitralstenose kann man in $13 \%$ der Fälle intraalveoläre Kalkdepots basal im Röntgenbild feststellen. Fördernd dabei sind eine länger bestehende pulmonale Hypertension sowie rezidivierend auftretende Lungenödeme. Da bei unserer Patientin die Mitralklappe deutlich verkalkt war, muß auch dies als Grund für die Verkalkungen in Betracht gezogen werden. Die Pleurosis calcarea ist in der Thoraxaufnahme in zwei Ebenen meist gut von intrapulmonalen Verkalkungen zu differenzieren. Häufig tritt sie nach Thoraxtrauma, -empyem und -erguß auf. Die Anamnese kann somit für diese DD wiederum richtungweisend sein.

Zur verbesserten Diagnostik empfehlen Romagnoli M. et al. die digitale Radiographie, welche zum Aufspüren von intrapulmonalen Verkalkungen sensitiver zu sein scheint als das konventionelle Thoraxröntgen.

Die CT ermöglicht durch genaue Dichtebestimmung die exakteste Diagnostik bei pulmonalen Verkalkungen und sollte in Zweifelsfällen bei Verdacht auf eine derartige Erkrankung als weiterführendes Diagnostikum angewendet werden.

T. Finkenzeller, K. Hill, J. Link, Regensburg 